\title{
Profiling microRNA from Brain by Microarray in a Transgenic Mouse Model of Alzheimer's Disease
}

\author{
Lin-lin Wang, ${ }^{1}$ Li Min, ${ }^{2}$ Qing-dong Guo, ${ }^{2}$ Jun-xia Zhang, ${ }^{1}$ Hai-lun Jiang, ${ }^{1}$ Shuai Shao, ${ }^{1}$ \\ Jian-guo Xing, ${ }^{3}$ Lin-lin Yin, ${ }^{4}$ Jiang-hong Liu, ${ }^{5}$ Rui Liu, ${ }^{1}$ and Shui-long Guo ${ }^{2}$ \\ ${ }^{1}$ Institute of Materia Medica, Chinese Academy of Medical Sciences \& Peking Union Medical College, Beijing 100050, China \\ ${ }^{2}$ Department of Gastroenterology, Beijing Friendship Hospital, Capital Medical University, \\ National Clinical Research Center for Digestive Disease, Beijing Key Laboratory for Precancerous Lesion of Digestive Disease, \\ Beijing 100050, China \\ ${ }^{3}$ Key Laboratory of Uighur Medicine of Xinjiang Uygur Autonomous Region, Xinjiang Institute of Materia Medica, \\ Urumqi 830004, China \\ ${ }^{4}$ Department of Neurology, Beijing Tiantan Hospital, Capital Medical University, Beijing 100050, China \\ ${ }^{5}$ Department of Neurology, Xuanwu Hospital, Capital Medical University, Beijing 100053, China
}

Correspondence should be addressed to Rui Liu; liurui@imm.ac.cn and Shui-long Guo; slong.guo@163.com

Received 31 May 2017; Accepted 9 August 2017; Published 19 September 2017

Academic Editor: Alessandro Martorana

Copyright (C) 2017 Lin-lin Wang et al. This is an open access article distributed under the Creative Commons Attribution License, which permits unrestricted use, distribution, and reproduction in any medium, provided the original work is properly cited.

\begin{abstract}
MicroRNAs (miRNAs) are small noncoding RNAs, which regulate numerous cell functions by targeting mRNA for cleavage or translational repression, and have been found to play an important role in Alzheimer's disease (AD). Our study aimed to identify differentially expressed miRNAs in AD brain as a reference of potential therapeutic miRNAs or biomarkers for this disease. We used amyloid precursor protein (APP) and presenilin 1 (PS1) double transgenic mice and age-matched wild-type (WT) littermates to determine the expression of miRNAs in the brain. MiRNAs were profiled by microarray, and differentially expressed miRNAs underwent target prediction and enrichment analysis. Microarray analysis revealed 56 differentially expressed miRNAs in AD mouse brain, which involved 39 miRNAs that were significantly upregulated and 19 that were downregulated at different ages. Among those miRNAs, a total of 11 miRNAs, including miR-342-3p, miR-342-5p, miR-376c-3p, and miR-301b-3p, were not only conserved in human but also predicted to have targets and signaling pathways closely related to the pathology of AD. In conclusion, in this study, differentially expressed miRNAs were identified in AD brain and proposed as biomarkers, which may have the potential to indicate AD progression. Despite being preliminary, these results may aid in investigating pathological hallmarks and identify effective therapeutic targets.
\end{abstract}

\section{Introduction}

Alzheimer's disease (AD), the main cause of dementia, is a progressive neurodegenerative disease that is characterized by extracellular senile plagues, intracellular neurofibrillary tangles (NFTs), and neuron loss $[1,2]$. Accumulation of the amyloid beta peptide $(\mathrm{A} \beta)$ within the brain, which is cleaved by $\beta$-secretase (BACE1) and $\gamma$-secretase from amyloid precursor protein (APP), is considered a major cause of AD [3]. Aggregation of $A \beta$ can disturb neurotransmission and cause synaptic impairment [4]. A $\beta$ oligomers trigger the immune system, leading to immune responses, such as the release of chemokines, proinflammatory cytokines, and complement factors. These will increase neuronal death and neuronal synapse loss [5-7]; therefore, the burden of $\mathrm{A} \beta$ in $\mathrm{AD}$ brain could be an indication of cognitive decline. Several factors have been confirmed to be connected with $\mathrm{AD}$, such as mitochondrial damage, synaptic loss, $A \beta$ accumulation, tau phosphorylation, neuroinflammation, and gene mutations [8-12]. Currently, no effective drugs or treatments exist that can prevent the progress of the disease, and most candidate drugs that targeted $\mathrm{A} \beta$ and tau failed during clinical or preclinical research. In addition, there are no noninvasive biomarkers of AD. Thus, microRNAs (miRNAs), which can be easily 
detected and are widely distributed, have become interesting in $\mathrm{AD}$ biomarker research.

MiRNAs are small single-stranded noncoding RNAs, consisting of about 20 nucleotides in length that are widely distributed in cells, blood, serum, and plasma, and regulate a myriad of cell functions [13-15]. Primary miRNA in the nucleus is processed into precursor-miRNA by the RNase III enzyme Drosha and transported to the cytoplasm where it is further processed by an enzyme Dicer to become mature miRNA $[12,16]$. Binding to the $3^{\prime}$ untranslated region $\left(3^{\prime}\right.$ UTR) of target mRNA will result in the formation of a silencing complex, in which miRNA regulates the translation process and inhibits the protein from being generated [17]. About $70 \%$ of all reported miRNAs can be found in the brains, and several of these miRNAs have been proven to be tightly connected with $\mathrm{AD}$ through binding to the $\beta$-site amyloid precursor protein cleaving enzyme BACE1 or other mRNAs in the amyloidogenic pathways to decrease overproduction of $\mathrm{A} \beta[18,19]$.

MiRNAs are reported to regulate cell functions, including $\mathrm{A} \beta$ processing, tau translating, apoptosis, inflammation, and cell cycle phase. Aberrant expression of miRNAs can affect the development and progression of $\operatorname{AD}[1,19,20]$. miR-29a, miR-29c, and miR-124 have been shown to interact with the $3^{\prime}$-UTR of BACE1 mRNA, and downregulation of miR-29a, miR-29c, and miR-124 in AD leads to overproduction of $\mathrm{A} \beta$ [21-23]. MiR-34a has also been reported to directly bind to tau mRNA and thereby changes the expression of miR-34a in $\mathrm{AD}$, which is important in the expression of tau protein [24].

MiRNA microassay provides an efficient tool for highthroughput and quantitative detection with excellent reproducibility. We detected 3100 capture probes, covering all human, mouse, and rat miRNAs annotated in miRBase 18.0, as well as all viral miRNAs related to these species in APP and presenilin 1 (PS1) double transgenic mouse brain compared with age-matched wild-type (WT) controls, and analyzed any changes to identify aberrant expression of miRNAs.

\section{Materials and Methods}

2.1. Animal and Tissue Preparation. Heterozygous APPswe/ PS1 $\Delta 9$ transgenic founder mice were purchased from Jackson Laboratory (Bar Harbor, ME, USA). Age-matched WT littermates were used as controls. All animals received care according to the Guide for the Care and Use of Laboratory Animals. Mice were maintained at a constant ambient temperature and a $12 \mathrm{~h}$ light/dark cycle, with free access to food and water. Mice were divided into the following groups: 1-month-old, 3-month-old, 6-month-old, and 9-month-old APP/PS1 mice (abbreviated to AD mice) and age-matched WT control mice. Each group contained 6 mice (three males and three females per group). From 3 mice, brains were collected and immediately frozen into liquid nitrogen. The remaining mice were used for additional gene and protein measurements. Both the initial division of mice into different groups and the subsequent selection of mice for gene and protein experiments were done randomly.

2.2. RNA Isolation. Total RNA was isolated using TRIzol (Invitrogen, Carlsbad, CA, USA) and purified using an RNeasy Mini Kit (QIAGEN, Dusseldorf, Germany) according to the manufacturer's instructions. RNA quality and quantity were measured using a Nanodrop spectrophotometer (ND-1000, Nanodrop Technologies, Wilmington, Delaware) and RNA integrity was determined by gel electrophoresis.

2.3. miRNA Labeling and Array Hybridization. RNA labeling and array hybridization were carried out according to Exiqon's manual (Exiqon, Vedbæk, Denmark). After preparation of the quality control, the miRCURY ${ }^{\mathrm{TM}} \mathrm{Hy}^{\mathrm{TM}} / \mathrm{Hy}^{\mathrm{TM}}$ Power labeling kit (Exiqon, Vedbæk, Denmark) was used for miRNA labeling according to the manufacturer's guidelines. A total of $1.0 \mu \mathrm{L}$ total RNA and $2.0 \mu \mathrm{L}$ water were combined with $1.0 \mu \mathrm{L}$ calf-intestinal alkaline phosphatase (CIP) (Exiqon, Vedbæk, Denmark) with the CIP buffer and incubated for $30 \mathrm{~min}$ at $37^{\circ} \mathrm{C}$, followed by incubation for $5 \mathrm{~min}$ at $95^{\circ} \mathrm{C}$. Then, a mixture, containing $3.0 \mu \mathrm{L}$ labeling buffer, $1.5 \mu \mathrm{L}$ fluorescent labels (Hy3), 2.0 $\mu \mathrm{L}$ DMSO, and $2.0 \mu \mathrm{L}$ labeling enzyme, was added and incubated for $1 \mathrm{~h}$ at $16^{\circ} \mathrm{C}$, followed by incubation for $15 \mathrm{~min}$ at $65^{\circ} \mathrm{C}$ to terminate the labeling. After stopping the labeling procedure, the Hy3labeled samples were hybridized onto the miRCURY LNA array (v.18.0) (Exiqon, Vedbæk, Denmark) according to array manual. The Hy3-labeled samples and $25 \mu \mathrm{L}$ hybridization buffer were denatured for $2 \mathrm{~min}$ at $95^{\circ} \mathrm{C}$ and hybridized to the microarray for $16 \mathrm{~h}-20 \mathrm{~h}$ at $56^{\circ} \mathrm{C}$ in a 12 -Bay Hybridization System (Hybridization System, Nimblegen Systems, Inc., Madison, WI, USA) following several times of washing using a wash buffer kit (Exiqon, Vedbæk, Denmark). Next, the slides were scanned by the Axon GenePix 4000B microarray scanner (Axon Instruments, Foster City, CA, USA).

2.4. Data Analysis. Scanned images were imported into GenePix Pro 6.0 software (Axon Instruments, Union City, CA, USA) for grid alignment and data analysis. Replicated miRNAs were averaged and miRNAs with an intensity $\geq 30$ in all samples were chosen for calculating the normalization factor using median normalization. Significantly differentially expressed miRNAs between two groups were identified by fold change and $P$ value, and fold change $\geq 2.0$ and $P$ value $\leq 0.05$ were considered significant. Differentially expressed miRNAs between two samples were filtered through fold change. Finally, hierarchical clustering was performed to identify distinguishable miRNA expression profiling among samples.

2.5. miRNA Target Prediction. For miRNA target prediction, the target prediction programs, involving TargetScan, miRDB, and miRanda, were used. The search was performed on the $3^{\prime}$-UTR regions of target mRNAs with a $P$ value of 0.05 defining the probability distribution of random matches set in the software with a minimum miRNA seed length of 7 $[25,26]$.

2.6. Functional Classification and Pathway Analysis. To further investigate the roles of miRNAs in the process of $\mathrm{AD}$, gene ontology (GO) and Kyoto Encyclopedia of Genes and Genomes (KEGG) pathway analysis were carried out using 

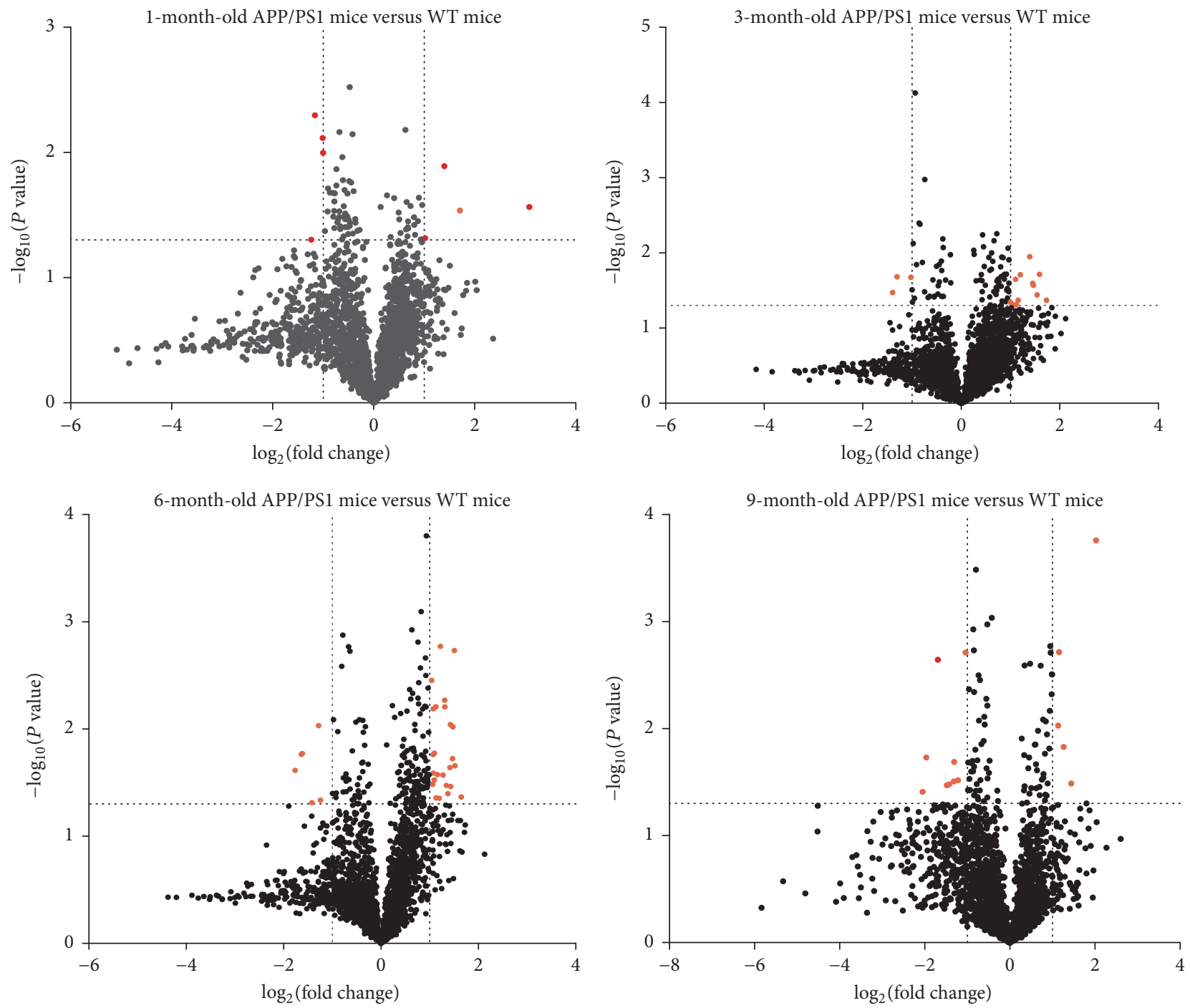

Figure 1: Scatter plot comparison of the fold changes and $P$ values of microarray experiments. The vertical lines correspond to 2 -fold up and down, respectively, and the horizontal line represents a $P$ value of 0.05 . The red point in the plot represents the differentially expressed miRNAs with statistical significance.

DAVID (https://david.ncifcrf.gov/). Targets were synthesized and intersections of miRDB, TargetScan, and miRanda databases of the 11 most significant changed miRNAs were taken, and then GO and KEGG analysis were performed.

\section{Results}

3.1. Significant Expression Changes of miRNAs in APP/PS1 Mice. To determine the miRNA levels in the brain, we evaluated the miRNAs in 1-month-old, 3-month-old, 6-monthold, and 9-month-old APP/PS1 mice and age-matched WT controls. APP/PS1 mice were analyzed by computational approaches and compared with age-matched WT controls (Figure 1). In contrast with the WT control group, 4 miRNAs were significantly upregulated and 4 were downregulated in the 1-month-old APP/PS1 mouse brain group. In addition, 11 miRNAs were upregulated and 3 downregulated in 3-monthold APP/PS1 mouse brain, 24 miRNAs were upregulated and 6 downregulated in 6-month-old APP/PS1 mouse brain, and 5 miRNAs were upregulated and 9 downregulated in 9month-old APP/PS1 mouse brain (Table 1). Among these differentially expressed miRNAs, miR-342-3p was consistently upregulated in 1-month-old, 6-month-old, and 9-month-old APP/PS1 mice, which suggests that miR-342-3p may play a role in the process of AD. Moreover, miR-342-5p was elevated in both 1-month-old and 3-month-old APP/PS1 mouse groups, suggesting that miR-342-5p may play a role in early stages of AD. Furthermore, levels of miR-2136, let-7f-5p, miR-431-5p, and miR-491-3p were higher in both 3-monthold and 6-month-old APP/PS1 mouse brain, indicating their potential involvement in the progression of $\mathrm{AD}$ (Figure 2).

3.2. Function and Pathway Analysis. To explore the potential functions of the significantly different expression of miRNAs, several bioinformatical analyses were performed. Figure 3 presents the clustering of the miRNAs per age group. For 
TABLE 1: Changes in expression of miRNAs in 1-month-old, 3-month-old, 6-month-old, and 9-month-old APP/PS1 mice as compared to age-matched WT controls.

\begin{tabular}{|c|c|c|c|c|c|}
\hline & Change & miRNA ID & miRNA name & Fold change & $P$ value \\
\hline \multirow{8}{*}{$\begin{array}{l}\text { 1-month-old APP/PS1 mice } \\
\text { versus WT control }\end{array}$} & \multirow{5}{*}{ Up } & 30681 & mmu-miR-376c-3p & 2.023 & 0.048 \\
\hline & & 42576 & mmu-miR-342-5p & 2.636 & 0.012 \\
\hline & & 42646 & $\mathrm{mmu}-\mathrm{miR}-878-3 \mathrm{p}$ & 8.470 & 0.027 \\
\hline & & 32884 & $\mathrm{mmu}-\mathrm{miR}-342-3 \mathrm{p}$ & 3.266 & 0.029 \\
\hline & & 146015 & mmu-miR-1940 & 0.446 & 0.005 \\
\hline & \multirow{3}{*}{ Down } & 168826 & mmu-miR-5624-5p & 0.500 & 0.010 \\
\hline & & 145692 & mmu-miR-499-3p & 0.420 & 0.047 \\
\hline & & 148259 & mmu-miR-3070a-5p/mmu-miR-3070b-5p & 0.497 & 0.008 \\
\hline \multirow{14}{*}{$\begin{array}{l}\text { 3-month-old APP/PS1 mice } \\
\text { versus WT control }\end{array}$} & \multirow{11}{*}{ Up } & 148453 & mmu-miR-3074-5p & 2.731 & 0.03 \\
\hline & & 17835 & mmu-miR-450a-5p & 2.621 & 0.011 \\
\hline & & 146004 & mmu-miR-2136 & 2.146 & 0.023 \\
\hline & & 148047 & mmu-miR-3058-3p & 2.761 & 0.027 \\
\hline & & 42576 & mmu-miR-342-5p & 2.066 & 0.049 \\
\hline & & 169158 & mmu-miR-344i & 2.163 & 0.049 \\
\hline & & 17752 & $m m u-l e t-7 f-5 p$ & 2.297 & 0.020 \\
\hline & & 46728 & mmu-miR-3059-3p & 3.315 & 0.043 \\
\hline & & 145836 & mmu-miR-218-2-3p & 2.910 & 0.036 \\
\hline & & 148074 & mmu-miR-539-3p & 3.002 & 0.019 \\
\hline & & 169301 & mmu-miR-3967 & 2.224 & 0.043 \\
\hline & \multirow{3}{*}{ Down } & 145705 & mmu-miR-431-5p & 0.405 & 0.021 \\
\hline & & 42595 & mmu-miR-291a-3p & 0.493 & 0.021 \\
\hline & & 147701 & mmu-miR-491-3p & 0.381 & 0.034 \\
\hline \multirow{30}{*}{$\begin{array}{l}\text { 6-month-old APP/PS1 mice } \\
\text { versus WT control }\end{array}$} & \multirow{30}{*}{ Down } & 146199 & mmu-miR-1961 & 2.696 & 0.034 \\
\hline & & 13485 & mmu-miR-10a-5p & 2.841 & 0.002 \\
\hline & & 17835 & mmu-miR-450a-5p & 2.483 & 0.006 \\
\hline & & 42712 & mmu-miR-742-5p & 2.692 & 0.009 \\
\hline & & 146004 & mmu-miR-2136 & 2.057 & 0.004 \\
\hline & & 42664 & mmu-miR-301b-3p & 2.668 & 0.023 \\
\hline & & 14189 & mmu-miR-302b-5p & 3.141 & 0.043 \\
\hline & & 145633 & mmu-let-7d-3p & 2.088 & 0.033 \\
\hline & & 19601 & mmu-miR-211-5p & 2.769 & 0.019 \\
\hline & & 11182 & mmu-miR-98-5p & 2.099 & 0.017 \\
\hline & & 32884 & mmu-miR-342-3p & 2.476 & 0.005 \\
\hline & & 27571 & mmu-miR-292-5p & 2.104 & 0.026 \\
\hline & & 46275 & mmu-miR-1251-5p & 2.329 & 0.002 \\
\hline & & 148017 & mmu-miR-743a-5p & 2.538 & 0.034 \\
\hline & & 10916 & mmu-miR-1a-3p & 2.140 & 0.017 \\
\hline & & 146137 & mmu-miR-133a-3p & 2.868 & 0.022 \\
\hline & & 148423 & mmu-miR-652-5p & 2.230 & 0.027 \\
\hline & & 17752 & mmu-let-7f-5p & 2.782 & 0.010 \\
\hline & & 11210 & mmu-miR-215-5p & 2.589 & 0.040 \\
\hline & & 146160 & mmu-miR-133b-3p & 2.285 & 0.044 \\
\hline & & 168586 & $m m u-m i R-34 a-5 p$ & 2.416 & 0.027 \\
\hline & & 42587 & mmu-miR-881-5p & 2.104 & 0.006 \\
\hline & & 46859 & mmu-miR-135a-2-3p & 2.192 & 0.044 \\
\hline & & 145914 & mmu-miR-135b-5p & 2.186 & 0.006 \\
\hline & & 42767 & mmu-miR-34c-3p & 0.412 & 0.009 \\
\hline & & 145705 & mmu-miR-431-5p & 0.326 & 0.017 \\
\hline & & 145637 & mmu-miR-187-3p & 0.294 & 0.024 \\
\hline & & 42929 & mmu-miR-25-5p & 0.374 & 0.049 \\
\hline & & 148276 & mmu-miR-3073a-3p & 0.322 & 0.017 \\
\hline & & 147701 & mmu-miR-491-3p & 0.422 & 0.046 \\
\hline
\end{tabular}


TABLE 1: Continued.

\begin{tabular}{|c|c|c|c|c|c|}
\hline & Change & miRNA ID & miRNA name & Fold change & $P$ value \\
\hline \multirow{14}{*}{$\begin{array}{l}\text { 9-month-old APP/PS1 mice } \\
\text { versus WT control }\end{array}$} & \multirow{5}{*}{ Up } & 30681 & mmu-miR-376c-3p & 2.407 & 0.015 \\
\hline & & 42494 & mmu-miR-712-3p & 2.721 & 0.033 \\
\hline & & 32884 & mmu-miR-342-3p & 4.074 & 0.0001 \\
\hline & & 169373 & mmu-miR-5626-5p & 2.195 & 0.009 \\
\hline & & 46297 & mmu-miR-3085-3p & 2.229 & 0.002 \\
\hline & \multirow{9}{*}{ Down } & 148605 & mmu-miR-128-2-5p & 0.430 & 0.030 \\
\hline & & 42595 & mmu-miR-291a-3p & 0.256 & 0.019 \\
\hline & & 148105 & mmu-miR-3106-3p & 0.359 & 0.034 \\
\hline & & 17866 & mmu-miR-331-5p & 0.403 & 0.020 \\
\hline & & 42625 & mmu-miR-299a-3p & 0.371 & 0.033 \\
\hline & & 148525 & mmu-miR-1964-5p & 0.241 & 0.039 \\
\hline & & 145757 & mmu-miR-33-3p & 0.487 & 0.002 \\
\hline & & 42621 & mmu-miR-879-3p & 0.401 & 0.031 \\
\hline & & 11038 & mmu-miR-299a-5p/mmu-miR-299b-5p & 0.310 & 0.002 \\
\hline
\end{tabular}

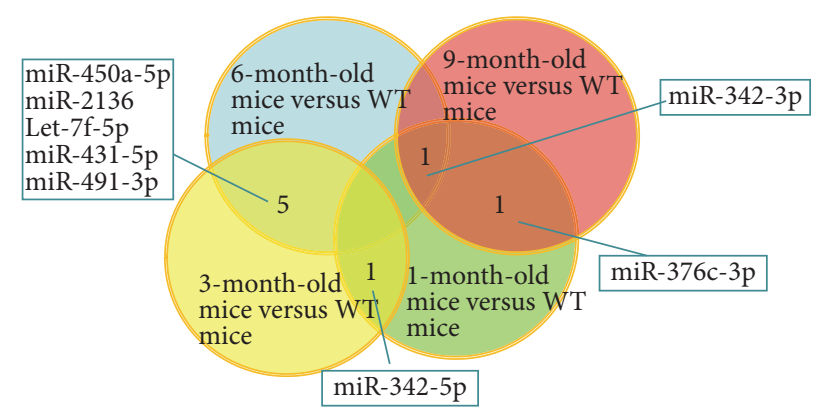

FIGURE 2: Venn diagram showing the number of miRNAs significantly changed and intersection of changed miRNAs between each age stage. Green areas show the number of changed miRNAs of 1-month-old mice. Yellow areas demonstrate the number of differentially expressed miRNAs in 3-month-old mice. Blue areas display the number of changed miRNAs in 6-month-old mice. Red areas show the number of differentially expressed miRNAs in 9month-old AD mice. The intersections indicate the miRNAs that changed in two groups or miRNAs that varied in three groups.

further analysis, we chose 11 evidently different miRNAs that were conserved between both human and mouse: miR-3423p, miR-342-5p, miR-376c-3p, miR-301b-3p, let-7f-5p, miR539-3p, miR-491-3p, miR-10a-5p, miR-98-5p, miR-652-5p, and miR-34a-5p.

GO and KEGG pathway analyses were performed to elucidate the biological function of the 11 selected miRNAs. GO terms covered 3 domains: molecular function, biological process, and cellular component. Figure 4 shows the top 12 significantly enriched GO terms at each domain. Moreover, in Figure 5, the 10 most significantly enriched pathways are shown that were mapped with KEGG pathway analysis: the Rapl signaling pathway, MAPK signaling pathway, signaling pathways regulating pluripotency of stem cells, endocytosis, TGF-beta signaling pathway, pathways in cancer, melanogenesis, choline metabolism in cancer, FoxO signaling pathway, and the Wnt signaling pathway.

3.3. Prediction Targets of miRNAs with Different Expressions in $A D$ Brain. To further elucidate the function of miRNAs and the roles of their target genes, we consulted the miRBase (http://www.mirbase.org/) and TargetScan (http://www .targetscan.org/) and summarized the miRNAs and their targets that tightly correspond to AD (Table 2).

Most of the miRNAs were targeted on APP processing and some of those directly combined with $3^{\prime}$-UTR of BACE1 mRNA. The downregulation of miR-1251-5p and miR-34a$5 p$ might alleviate cognitive deficit by increasing the activity of disintegrin and metalloprotease 10 (ADAM10), a major $\alpha$ secretase in brain that is capable of attenuating the production of $\mathrm{A} \beta$ by cleavage of APP, resulting in soluble APP $\alpha$ [27]. MiR-342-5p, miR-3058-3p, let-7f-5p, miR-1961, miR-301b3p, miR-98-5p, miR-1251-5p, miR-215-5p, miR-881-5p, miR$135 a-2-3 p$, and miR-33-3p may regulate the expression of insulin-like growth factor 1 (IGF1) or insulin-like growth factor 2 (IGF2), two molecules that could rescue behavior and memory deficits via lowering $\mathrm{A} \beta$ levels $[28,29]$. The other miRNAs may participate in the phosphorylation of tau, which was the main cause of NFTs. Tau phosphorylation is mainly regulated by glycogen synthase kinase $3 \beta$ (GSK $3 \beta$ ) while Type $2 \mathrm{~A}$ protein phosphatase (PP2A) modulates the dephosphorylation of tau protein $[30,31]$. miR-376c-3p and miR-342-3p were predicted to affect tau phosphorylation by directly targeting the $3^{\prime}$-UTR of PP2A. Phosphatase and tensin homolog deleted on chromosome ten (PTEN) has been shown to inactivate the phosphatidylinositol 3-kinase (PI3K)/Akt signaling pathway and the absence of PTEN elevates tau aggregation $[32,33]$. Several miRNAs derived from our microarray analysis targeted PTEN, such as miR-376c-3p, miR-342-3p, let-7f-5p, miR-10a-5p, miR-301b-3p, miR-98-5p, miR-1251-5p, and miR-34a-5p.

\section{Discussion}

$\mathrm{AD}$ is a common form of dementia, with a fast growing morbidity with the speeding aging of the population all over the word. AD patients suffer not only from cognitive dysfunction, such as impairment of memory, visuospatial, language, and executive functions, but also from other psychological symptoms including depression [34]. Therefore, $\mathrm{AD}$ patients will gradually lose the ability to take care of 


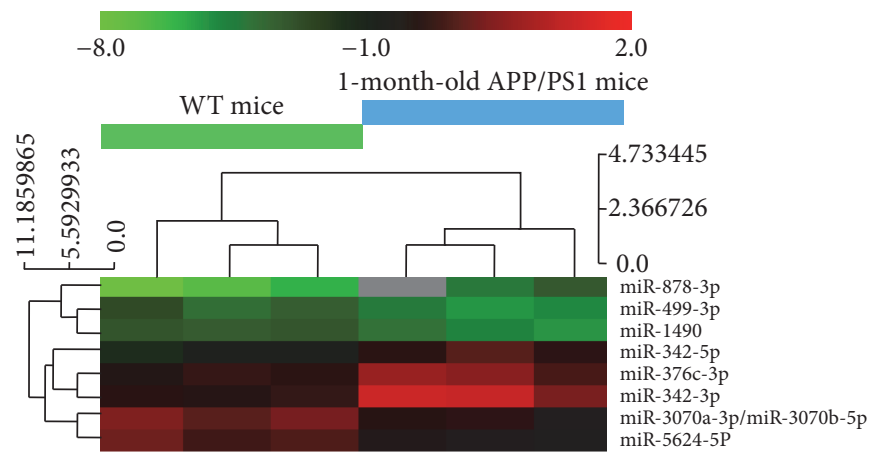

(a)

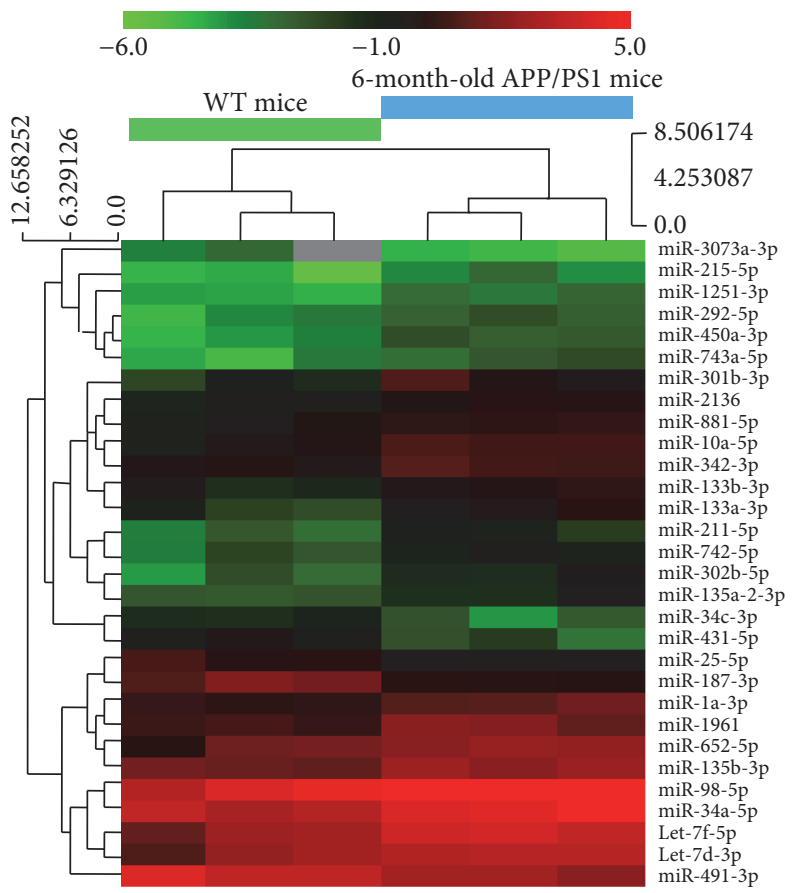

(c)

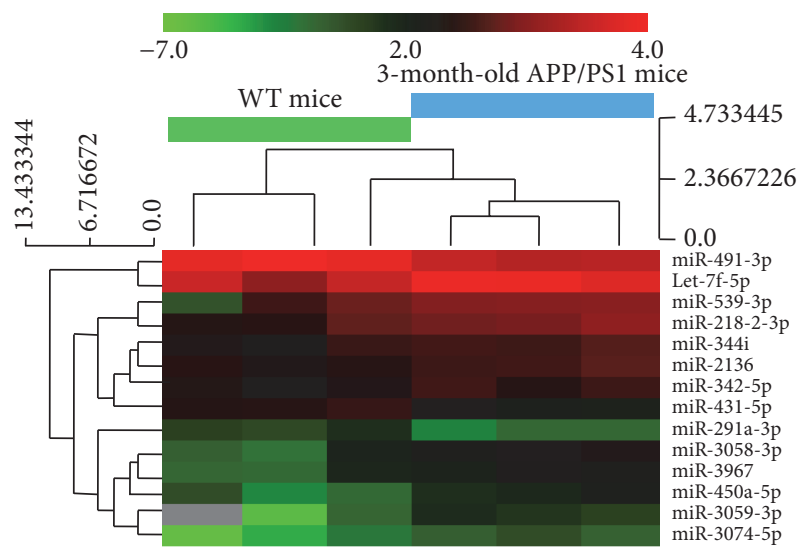

(b)

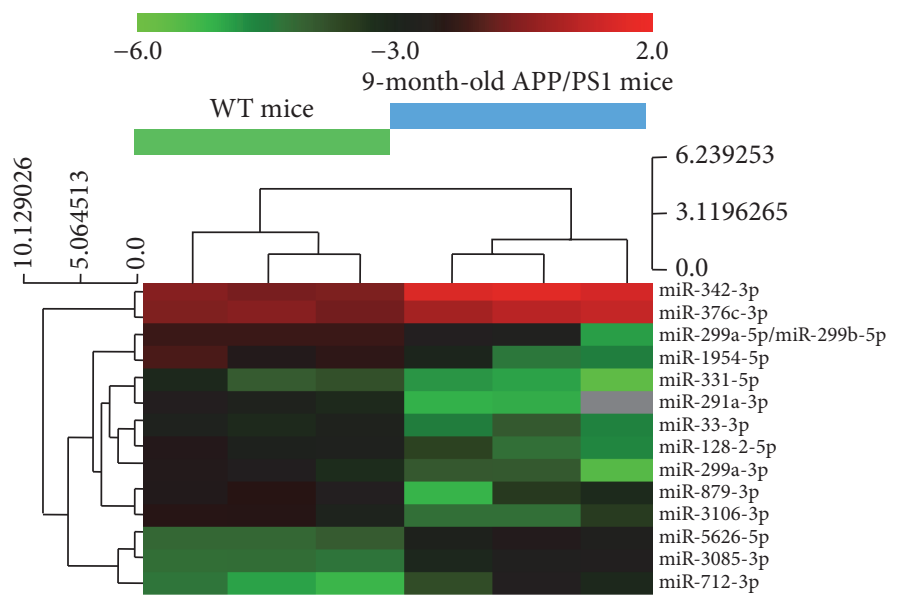

(d)

FIgURE 3: Two-way hierarchical clustering of miRNAs and brain samples. Each row represents a miRNA and each column represents a brain sample. Wide-type control mice are indicated as WT mice. Different age-stage AD mice are shown as 1-month-old (a), 3-month-old (b), 6-month-old (c), and 9-month-old (d) APP/PS1 mice. Clustering tree is shown on the left, and the sample clustering tree appears at the top. The color scale shown at the top illustrates the relative expression level of a miRNA in a certain age group. Red color represents a high relative expression level and a green color represents a low relative expression level.

themselves and suffer from both mental and psychological distress [35]. However, there are neither effective therapeutic strategies nor early diagnostic biomarkers for the treatment of $\mathrm{AD}$. In addition, in most patients, $\mathrm{AD}$ is hardly recognized in the early stages of the disease, and therefore the therapeutic opportunity is often missed because of the delay in diagnosis. miRNAs that regulate posttranscription of proteins have been reported to specifically change in the process of $\mathrm{AD}$ and can be easily detected by microarray analysis or RT-PCR.
Therefore, miRNAs could potentially serve as noninvasive biomarkers and therapeutic targets of $\mathrm{AD}$ [36].

To confirm the variation in miRNA expression at different stages of $\mathrm{AD}$, we chose 1-month-old, 3-month-old, 6-monthold, and 9-month-old AD mice, which were used for microarray analysis. Several differentially expressed miRNAs were found in different stages of AD. Of these different miRNAs, 28 were conserved between human and mouse brain: miR-376c3p, miR-342-5p, miR-342-3p, miR-499-3p, miR-450a-5p, 
GO biological process category

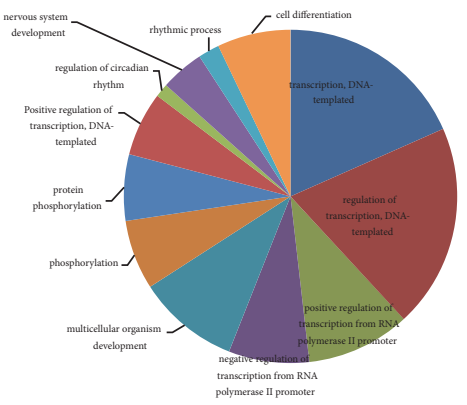

GO cellular component category

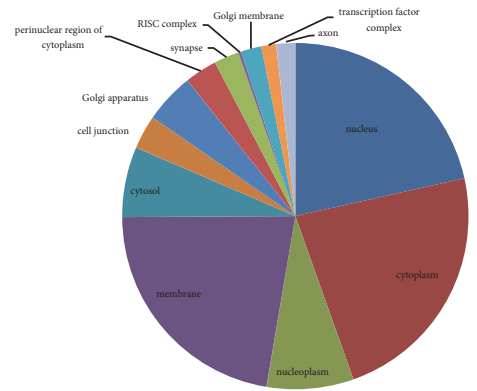

GO molecular function category

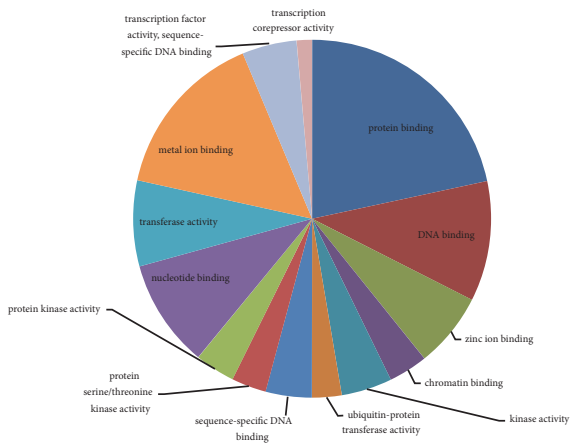

FIGURE 4: GO enrichment analysis of targets of selected miRNAs carried out with DAVID.

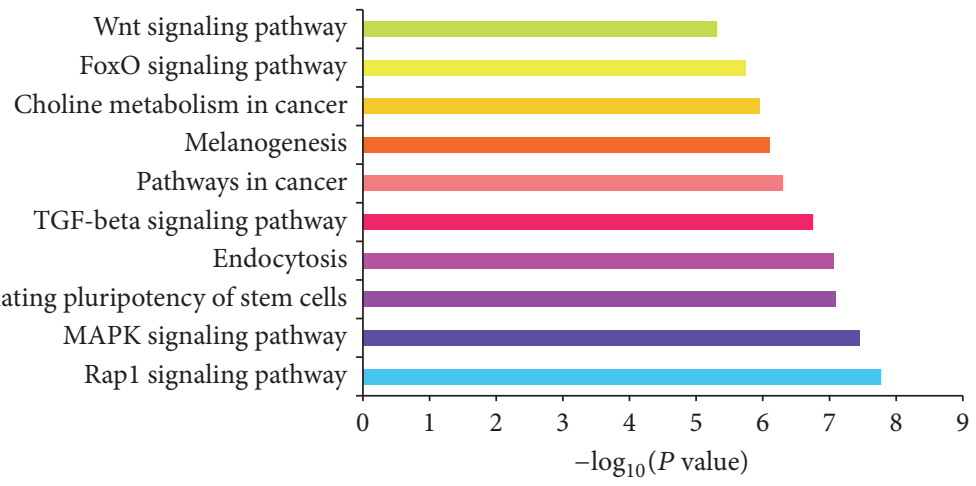

FIGURE 5: Top 10 significantly enriched pathways of selected 11 miRNAs analyzed with KEGG. 
TABLE 2: Significantly changed miRNAs and their predicted targets.

\begin{tabular}{lcc}
\hline miRNA ID & miRNA name & Target \\
\hline 42576 & mmu-miR-342-5p & IGF2, BACE1 \\
148047 & mmu-miR-3058-3p & IGF2 \\
17752 & mmu-let-7f-5p & IGF1, PTEN \\
148074 & mmu-miR-539-3p & GSK3 $\beta$ \\
42595 & mmu-miR-291a-3p & APP \\
147701 & mmu-miR-491-3p & BACE1, BDNF \\
146199 & mmu-miR-1961 & IGF1, IGF2 \\
13485 & mmu-miR-10a-5p & BDNF, PTEN \\
42664 & mmu-miR-301b-3p & IGF1, LDLR, NAV3, PTEN \\
19601 & mmu-miR-211-5p & BDNF \\
11182 & mmu-miR-98-5p & BACE1, IGF2, PTEN \\
46275 & mmu-miR-1251-5p & IGF1, APBB2, PTEN, ADAM10 \\
148423 & mmu-miR-652-5p & BACE1 \\
11210 & mmu-miR-215-5p & IGF1 \\
42587 & mmu-miR-34a-5p & PTEN, ADAM10 \\
46859 & mmu-miR-881-5p & IGF1, APP, IGF2, \\
3068157 & mmu-miR-135a-2-3p & IGF2 \\
32884 & mmu-miR-33-3p & IGF1 \\
\hline
\end{tabular}

let-7f-5p, miR-218-2-3p, miR-539-3p, miR-431-5p, miR-4913p, miR-10a-5p, miR-301b-3p, miR-302b-5p, let-7d-3p, miR211-5p, miR-98-5p, miR-1251-5p, miR-133a-3p, miR-652-5p, miR-215-5p, miR-34a-5p, miR-135b-5p, miR-34c-3p, miR187-3p, miR-25-5p, miR-376c-3p, miR-128-2-5p, and miR-331$5 \mathrm{p}$. Considering a probe signal of over 100 as abundance, eleven of the 28 miRNAs (miR-342-3p, miR-342-5p, miR376c-3p, miR-301b-3p, let-7f-5p, miR-539-3p, miR-491-3p, miR-10a-5p, miR-98-5p, miR-652-5p, and miR-34a-5p) were shown to have targets that are tightly related to $\mathrm{AD}$ and could easily be detected. Among these 11 miRNAs, only miR-4913 p was significantly downregulated, while the others were prominently upregulated.

In these differentially expressed miRNAs, miR-342$3 \mathrm{p}$ inhibited cell proliferation, migration, and invasion in osteosarcoma, gliomas, and other cancers [37-39]. miR-491 plays a role in different types of cancers, such as glioblastoma and tongue cancer $[40,41]$. However, no reports have been published that linked the two miRNAs to AD. To our knowledge, this is the first study to identify the potential effects of miR-342-3p, miR-491-3p, miR-539-3p, miR-376c-3p, miR$10 \mathrm{a}-5 \mathrm{p}$, and miR-652-5p in the progression of AD. miR$342-5 p$ was reported to manage neuron stem proliferation and differentiation by targeting Notch signaling [42]. It was shown to be upregulated in $\mathrm{AD}$ mouse brain and predicted to hamper the function of axon initial segment by decreasing the expression of ankyrin G. Predicted to target BACE1 and IGF2, miR-342-5p may play a significant role in $A D$. In our findings, let-7f-5p levels were higher in both 3-monthold and 6-month-old APP/PS1 mouse brain, compared to older mice. Unlike our results, let-7f-5p was not significantly different in plasma from $\mathrm{AD}$ patients and healthy controls
[43]. Thus, we speculate that let-7f-5p may have a different tissue distribution and developmental expression in brain and plasma. miR-301b promoted cell migration and invasion in bladder cancer through downregulating the expression of PTEN, which suggests that miR-301b-3p might target the $3^{\prime}$-UTR of PTEN and regulate the aggregation of tau [44]. miR-98-5p has been proven to regulate the expression of $\mathrm{A} \beta$ and might be a novel target of $\mathrm{AD}$ [45]. Consistent with the results of a previous study, which illustrated the alteration in plasma samples from $\mathrm{AD}$ patients [46], miR$34 a-5 p$ showed remarkable upregulation in 6-month-old AD mouse brain and was predicted to be positively connected to the APP process. Therefore, miR-34a-5p could be a potential biomarker for AD. Among the targets of the differentially expressed miRNAs (Table 2), IGF1 and IGF2 were predicted to be the proteins that are affected by a large scale of those differentially expressed miRNAs, such as miR-1961, let-7f-5p, and miR-135a-2-3p. IGF1 and IGF2 are receptors of insulin, and insulin plays an important role in $\mathrm{AD}$ by activating the PI3K/Akt signaling pathway and Ras/mitogen activated kinase (MAPK) cascades [47]. Taken together, miRNAs alleviate $\mathrm{AD}$ impairment by modulating the expression of IGF1 and IGF2, and therefore the insulin pathway may be a potential target of AD.

To deepen our understanding of these differentially expressed miRNAs, we performed two types of enrichment analyses: GO and KEGG. In this study, the analyses are focused upon the differential expression characteristics of those miRNAs involved in the pathological pathways of $\mathrm{AD}$, to better understand the underlying mechanisms of miRNA and predicted target transductions in this disease. These bioinformatics analyses enabled us to identify many putative 
signaling pathways in $\mathrm{AD}$ pathology in which the differentially expressed miRNAs are involved. These pathological pathways cover the nerve system development process, synapse component, and MAPK signaling pathways. Longterm potentiation (LTP) with 16 counts of total predicted targets and axon guidance with 22 counts also showed significant enrichment, which is not reflected in Figure 4. Combined with the target prediction analysis, we suggest that the 11 selected miRNAs may play a role in $\mathrm{AD}$ by regulating the activity in neuronal molecular events that are related to amyloidogenic processes, tau aggregation, and neuron injury.

Other than performing experiments on blood and cerebrospinal fluid (CSF), we specifically explored the variation of miRNAs in brain, which directly reflected the changes of miRNA in AD brain. Moreover, we explored four age groups, including early stage, medium stage, and late stage of $\mathrm{AD}$, and analyzed the variation of miRNA expression in these four groups. Although it is challenging to compare our results to the results of other studies in which different tissues and species were used, several miRNAs, including let-7f-5p, miR342-5p, and miR-98-5p, showed a similar trend in human blood and CSF $[48,49]$. In addition, some differences were found between those results; for example, the level of miR342-3p was downregulated in AD patient plasma samples, while in our study it was significantly elevated in APP/PS1 mouse brain of 1-month-old, 6-month-old, and 9-month-old mice [46].

miRNA microassay achieved high-throughput screening of miRNAs, and 11 significantly changed miRNAs conserved between human and mouse brain were found in $\mathrm{AD}$ brain. Moreover, the alterations of these miRNAs indicate a different development process of AD as indicated by miRNA expression levels at different ages. Among these miRNAs, miR-342$3 \mathrm{p}$ was upregulated from 1-month-old to 9-month-old AD mice, suggesting that it may play a critical role in the entire $\mathrm{AD}$ process. miR-342-5p was upregulated in brain tissue of 1-month-old and 6-month-old mice, indicating that it is effective at early onset and therefore may act as a specific early biomarker of AD. miR-2136, let-7f-5p, miR-431-5p, and miR491-3p were upregulated in both 3-month-old and 6-monthold $\mathrm{AD}$ mice, implying their role in the development of AD. Combined with subsequent target prediction and enrichment analysis, the 11 miRNAs were predicted to be closely related to the molecular events of the amyloidogenic process, tau phosphorylation, and MAPK and TGF- $\beta$ signaling pathways. Although these miRNAs need to be confirmed due to clearing of false-positive reactions, these findings illustrate that intervention of these miRNAs may affect the signaling transductions from transcriptional to posttranscriptional events and play a role in different processes of $\mathrm{AD}$. In the near future, clinical samples, such as plasma and CSF, are required to detect miRNA levels, targets, and signaling pathways predicted in our study. Thus, our study is a link between the microarray results translating from an animal model to patients who are diagnosed with $\mathrm{AD}$ and have developed cognitive deficits with pathological alterations of extracellular $\mathrm{A} \beta$ deposition and intracellular NFTs.

\section{Conclusions}

In summary, this study was also the first miRNA microarray using different-stage AD brain tissue, and through bioinformatics analysis, eleven miRNAs were shown to have plentiful altered expressions and to be conserved in evolution with possible target correlation to $\mathrm{AD}$ and suggested to act as "markers" to distinguish the pathological changes of this disease. Our findings provided a clue for understanding the pathological hallmarks and looking for effective therapeutic targets in the miRNA-mRNA level of AD.

\section{Conflicts of Interest}

The authors declare that they have no conflicts of interest regarding the publication of this paper.

\section{Acknowledgments}

This study was supported by the National Natural Science Foundation of China (nos. 81673411 and 81670474), Key Laboratory of Uighur Medicine Foundation of Xinjiang Uygur Autonomous Region (2015KL005), and CAMS Innovation Fund for Medical Science (2017-I2M-1-016).

\section{References}

[1] J. Satoh, "Molecular network of microRNA targets in Alzheimer's disease brains," Experimental Neurology, vol. 235, no. 2, pp. 436-446, 2012.

[2] P. Scheltens, K. Blennow, M. M. Breteler et al., "Alzheimer's disease," The Lancet, 2016.

[3] J. D. Barritt and J. H. Viles, "Truncated amyloid- $\beta(11-40 / 42)$ from Alzheimer disease binds $\mathrm{Cu} 2+$ with a femtomolar affinity and influences fiber assembly," Journal of Biological Chemistry, vol. 290, no. 46, pp. 27791-27802, 2015.

[4] S. Forner, D. Baglietto-Vargas, A. C. Martini, L. Trujillo-Estrada, and F. M. LaFerla, "Synaptic Impairment in Alzheimer's Disease: A Dysregulated Symphony," Trends in Neurosciences, vol. 40, no. 6, pp. 347-357, 2017.

[5] N. Liu, Y. Zhuang, Z. Zhou, J. Zhao, Q. Chen, and J. Zheng, "NF- $\kappa \mathrm{B}$ dependent up-regulation of TRPC6 by $\mathrm{A} \beta$ in BV-2 microglia cells increases COX-2 expression and contributes to hippocampus neuron damage," Neuroscience Letters, vol. 651, pp. 1-8, 2017.

[6] D. Raman, S.-Z. Milatovic, D. Milatovic, R. Splittgerber, G.H. Fan, and A. Richmond, "Chemokines, macrophage inflammatory protein- 2 and stromal cell-derived factor- $1 \alpha$, suppress amyloid $\beta$-induced neurotoxicity," Toxicology and Applied Pharmacology, vol. 256, no. 3, pp. 300-313, 2011.

[7] J. Daly IV and G. J. Kotwal, "Pro-inflammatory complement activation by the $\mathrm{A} \beta$ peptide of Alzheimer's disease is biologically significant and can be blocked by vaccinia virus complement control protein," Neurobiology of Aging, vol. 19, no. 6, pp. 619-627, 1998.

[8] X. Wang, W. Wang, L. Li, G. Perry, H. G. Lee, and X. Zhu, "Oxidative stress and mitochondrial dysfunction in Alzheimer's disease," Biochimica et Biophysica Acta, vol. 1842, no. 8, pp. 1240-1247, 2013. 
[9] J. Kim, H. Yoon, J. Basak, and J. Kim, "Apolipoprotein E in synaptic plasticity and Alzheimer's disease: potential cellular and molecular mechanisms," Molecules and cells, vol. 37, no. 11, pp. 767-776, 2014.

[10] M. Gold and J. El Khoury, " $\beta$-amyloid, microglia, and the inflammasome in Alzheimer's disease," Seminars in Immunopathology, vol. 37, no. 6, pp. 607-611, 2015.

[11] C. H. Latta, H. M. Brothers, and D. M. Wilcock, "Neuroinflammation in Alzheimer's disease; A source of heterogeneity and target for personalized therapy," Neuroscience, vol. 302, pp. 103111, 2015.

[12] E. Bagyinszky, Y. C. Youn, S. S. A. An, and S. Kim, “The genetics of Alzheimer's disease," Clinical Interventions in Aging, vol. 9, pp. 535-551, 2014.

[13] L. A. Smyth, D. A. Boardman, S. L. Tung, R. Lechler, and G. Lombardi, "MicroRNAs affect dendritic cell function and phenotype," Immunology, vol. 144, no. 2, pp. 197-205, 2015.

[14] C. K. Sen and S. Ghatak, "miRNA Control of Tissue Repair and Regeneration," American Journal of Pathology, vol. 185, no. 10, article no. 2054, pp. 2629-2640, 2015.

[15] S. Kumar and P. H. Reddy, "Are circulating microRNAs peripheral biomarkers for Alzheimer's disease?" Biochimica et Biophysica Acta - Molecular Basis of Disease, vol. 1862, no. 9, pp. 1617-1627, 2016.

[16] L. Fabris, Y. Ceder, A. M. Chinnaiyan et al., "The Potential of MicroRNAs as Prostate Cancer Biomarkers," European Urology, vol. 70, no. 2, pp. 312-322, 2016.

[17] L. Qiu, W. Zhang, E. K. Tan, and L. Zeng, "Deciphering the function and regulation of microRNAs in Alzheimer's disease and Parkinson's disease," ACS Chemical Neuroscience, vol. 5, no. 10, pp. 884-894, 2014.

[18] Y. Deng, Y. Ding, and D. Hou, "Research status of the regulation of miRNA on BACE1," International Journal of Neuroscience, vol. 124, no. 7, pp. 474-477, 2014.

[19] C. Delay, W. Mandemakers, and S. S. Hébert, "MicroRNAs in Alzheimer's disease," Neurobiology of Disease, vol. 46, no. 2, pp. 285-290, 2012.

[20] L. Tan, J.-T. Yu, N. Hu, and L. Tan, "Non-coding RNAs in Alzheimer's disease," Molecular Neurobiology, vol. 47, no. 1, pp. 382-393, 2013.

[21] S. S. Hébert, K. Horré, L. Nicolaï et al., "Loss of microRNA cluster miR-29a/b-1 in sporadic Alzheimer's disease correlates with increased BACE1/ $\beta$-secretase expression," Proceedings of the National Academy of Sciences of the United States of America, vol. 105, no. 17, pp. 6415-6420, 2008.

[22] Y. Zong, H. Wang, W. Dong et al., "miR-29c regulates BACE1 protein expression," Brain Research, vol. 1395, pp. 108-115, 2011.

[23] M. Fang, J. Wang, X. Zhang et al., "The miR-124 regulates the expression of BACE1/ $\beta$-secretase correlated with cell death in Alzheimer's disease," Toxicology Letters, vol. 209, no. 1, pp. 94105, 2012.

[24] J. R. Dickson, C. Kruse, D. R. Montagna, B. Finsen, and M. S. Wolfe, "Alternative polyadenylation and miR-34 family members regulate tau expression," Journal of Neurochemistry, vol. 127, no. 6, pp. 739-749, 2013.

[25] B. P. Lewis, C. B. Burge, and D. P. Bartel, "Conserved seed pairing, often flanked by adenosines, indicates that thousands of human genes are microRNA targets," Cell, vol. 120, no. 1, pp. 15-20, 2005.

[26] A. Krek, D. Grün, M. N. Poy et al., "Combinatorial microRNA target predictions," Nature Genetics, vol. 37, no. 5, pp. 495-500, 2005.
[27] L. M. Bekris, F. Lutz, G. Li et al., "ADAM10 expression and promoter haplotype in Alzheimer's disease," Neurobiology of Aging, vol. 33, no. 9, pp. 2229-e9, 2012.

[28] M. Pascual-Lucas, S. Viana da Silva, M. Di Scala et al., "Insulinlike growth factor 2 reverses memory and synaptic deficits in APP transgenic mice," EMBO Molecular Medicine, vol. 6, no. 10, pp. 1246-1262, 2014.

[29] E. Carro, J. L. Trejo, T. Gomez-Isla, D. LeRoith, and I. TorresAleman, "Serum insulin-like growth factor I regulates brain amyloid- $\beta$ levels," Nature Medicine, vol. 8, no. 12, pp. 1390-1397, 2002.

[30] L. Martin, X. Latypova, C. M. Wilson, A. Magnaudeix, M.-L. Perrin, and F. Terro, "Tau protein phosphatases in Alzheimer's disease: the leading role of PP2A," Ageing Research Reviews, vol. 12, no. 1, pp. 39-49, 2013.

[31] J. J. Heinisch and R. Brandt, "Signaling pathways and posttranslational modifications of tau in Alzheimer's disease: the humanization of yeast cells," Microbial Cell, vol. 3, no. 4, pp. 135146, 2016.

[32] L. Tan, X. Chen, W. Wang et al., "Pharmacological inhibition of PTEN attenuates cognitive deficits caused by neonatal repeated exposures to isoflurane via inhibition of NR2B-mediated tau phosphorylation in rats," Neuropharmacology, vol. 114, pp. 135145, 2017.

[33] Y. Sonoda, H. Mukai, K. Matsuo et al., "Accumulation of tumorsuppressor PTEN in Alzheimer neurofibrillary tangles," Neuroscience Letters, vol. 471, no. 1, pp. 20-24, 2010.

[34] B. Yang, H. Yu, M. Xing, R. He, R. Liang, and L. Zhou, “The relationship between cognition and depressive symptoms, and factors modifying this association, in Alzheimer's disease: A multivariate multilevel model," Archives of Gerontology and Geriatrics, vol. 72, pp. 25-31, 2017.

[35] J. Lee, K. J. Lee, and H. Kim, “Gender differences in behavioral and psychological symptoms of patients with Alzheimer's disease," Asian Journal of Psychiatry, vol. 26, pp. 124-128, 2017.

[36] V. Van Giau and S. S. A. An, "Emergence of exosomal miRNAs as a diagnostic biomarker for Alzheimer's disease," Journal of the Neurological Sciences, vol. 360, pp. 141-152, 2016.

[37] S. Zhang, L. Liu, Z. Lv, Q. Li, W. Gong, and H. Wu, "MicroRNA342-3p Inhibits the Proliferation, Migration, and Invasion of Osteosarcoma Cells By Targeting Astrocyte-Elevated Gene-1 (AEG-1)," Oncology Research Featuring Preclinical and Clinical Cancer Therapeutics.

[38] W. Zhang, Y. Bi, J. Li et al., "Long noncoding RNA FTX is upregulated in gliomas and promotes proliferation and invasion of glioma cells by negatively regulating miR-342-3p," Laboratory Investigation, vol. 97, no. 4, pp. 447-457, 2017.

[39] H. Xu, T. Cai, G. N. Carmona, L. Abuhatzira, and A. L. Notkins, "Small cell lung cancer growth is inhibited by miR-342 through its effect of the target gene IA-2," Journal of Translational Medicine, vol. 14, no. 1, article no. 278, 2016.

[40] G. Zheng, X. Jia, C. Peng et al., "The miR-491-3p/mTORC2/ FOXO1 regulatory loop modulates chemo-sensitivity in human tongue cancer," Oncotarget, vol. 6, no. 9, pp. 6931-6943, 2015.

[41] X. Li, Y. Liu, K. J. Granberg et al., "Two mature products of MIR-491 coordinate to suppress key cancer hallmarks in glioblastoma," Oncogene, vol. 34, no. 13, pp. 1619-1628, 2015.

[42] F. Gao, Y.-F. Zhang, Z.-P. Zhang et al., "miR-342-5p Regulates Neural Stem Cell Proliferation and Differentiation Downstream to Notch Signaling in Mice," Stem Cell Reports, vol. 8, no. 4, pp. 1032-1045, 2016. 
[43] N. Hara, M. Kikuchi, A. Miyashita et al., "Serum microRNA miR-501-3p as a potential biomarker related to the progression of Alzheimer's disease," Acta Neuropathologica Communications, vol. 5, no. 1, 2017.

[44] H. Egawa, K. Jingushi, T. Hirono et al., "The miR-130 family promotes cell migration and invasion in bladder cancer through FAK and Akt phosphorylation by regulating PTEN," Scientific Reports, vol. 6, Article ID 20574, 2016.

[45] Q. Li, X. Li, L. Wang, Y. Zhang, and L. Chen, "miR-98-5p Acts as a Target for Alzheimer's Disease by Regulating A $\beta$ Production Through Modulating SNX6 Expression," Journal of Molecular Neuroscience, vol. 60, no. 4, pp. 413-420, 2016.

[46] M. Cosín-Tomás, A. Antonell, A. Lladó et al., "Plasma miR-34a$5 p$ and miR-545-3p as Early Biomarkers of Alzheimer's Disease: Potential and Limitations," Molecular Neurobiology, pp. 1-13, 2016.

[47] K. D. Niswender, C. D. Morrison, D. J. Clegg et al., "Insulin activation of phosphatidylinositol 3-kinase in the hypothalamic arcuate nucleus: A key mediator of insulin-induced anorexia," Diabetes, vol. 52, no. 2, pp. 227-231, 2003.

[48] J.-I. Satoh, Y. Kino, and S. Niida, "MicroRNA-Seq data analysis pipeline to identify blood biomarkers for alzheimer's disease from public data," Biomarker Insights, vol. 2015, no. 10, pp. 21-31, 2015.

[49] G. Lugli, A. M. Cohen, D. A. Bennett et al., "Plasma exosomal miRNAs in Persons with and without Alzheimer disease: altered expression and prospects for biomarkers," PLOS ONE, vol. 10, no. 10, Article ID e0139233, 2015. 

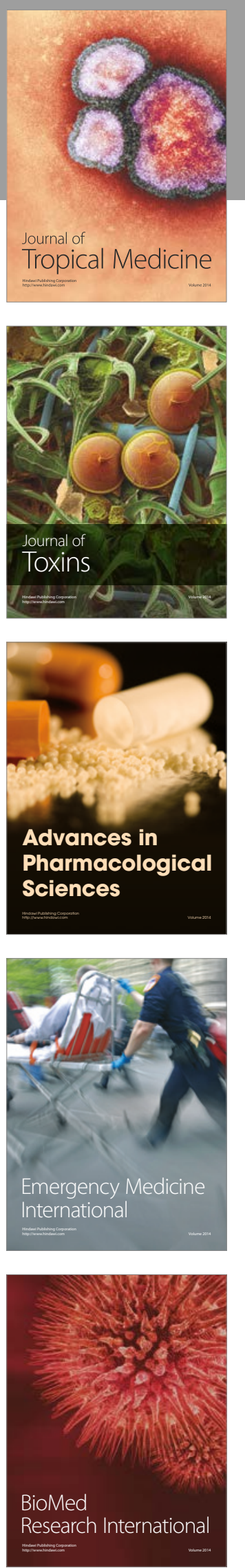
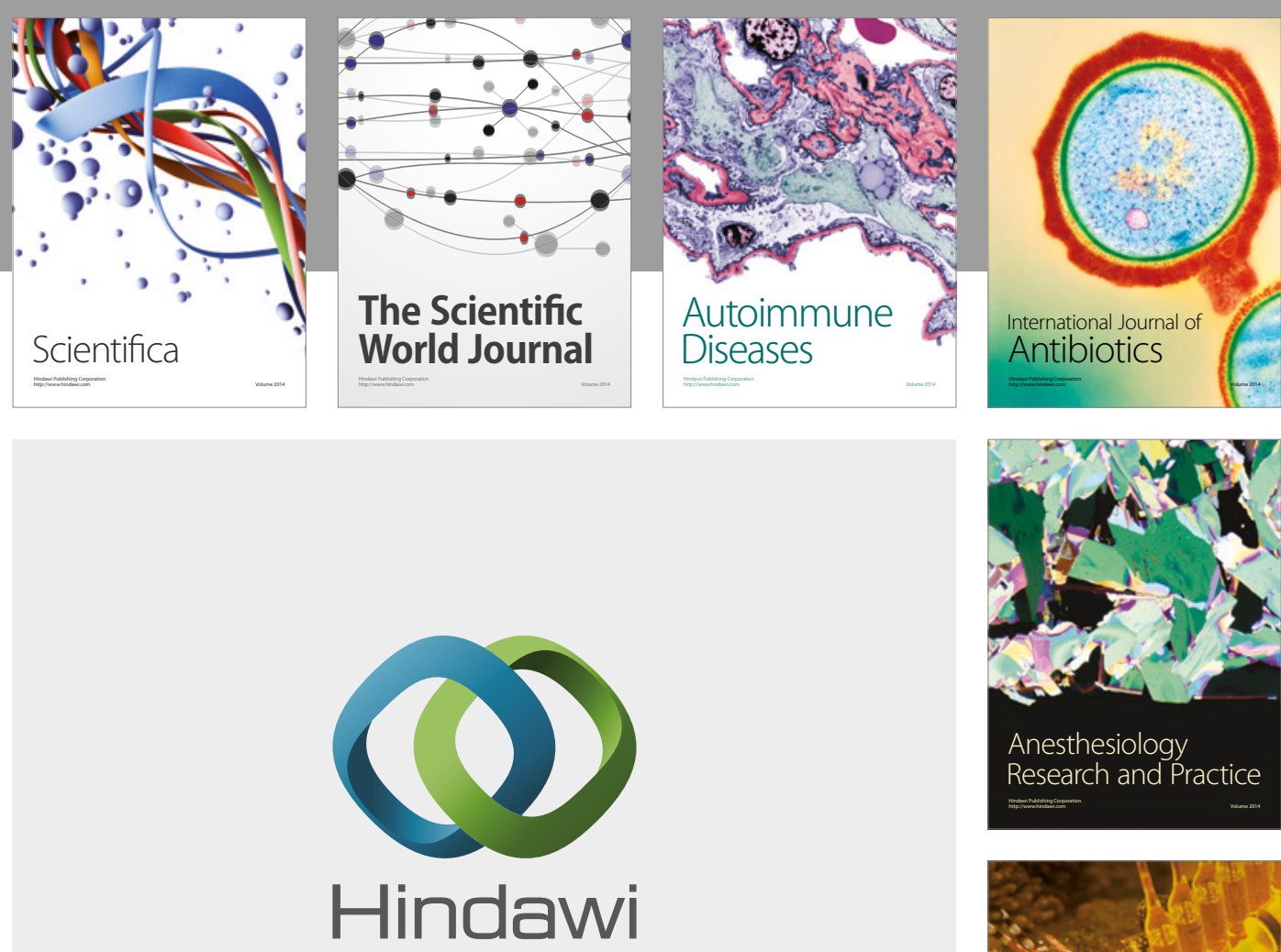

Submit your manuscripts at

https://www.hindawi.com
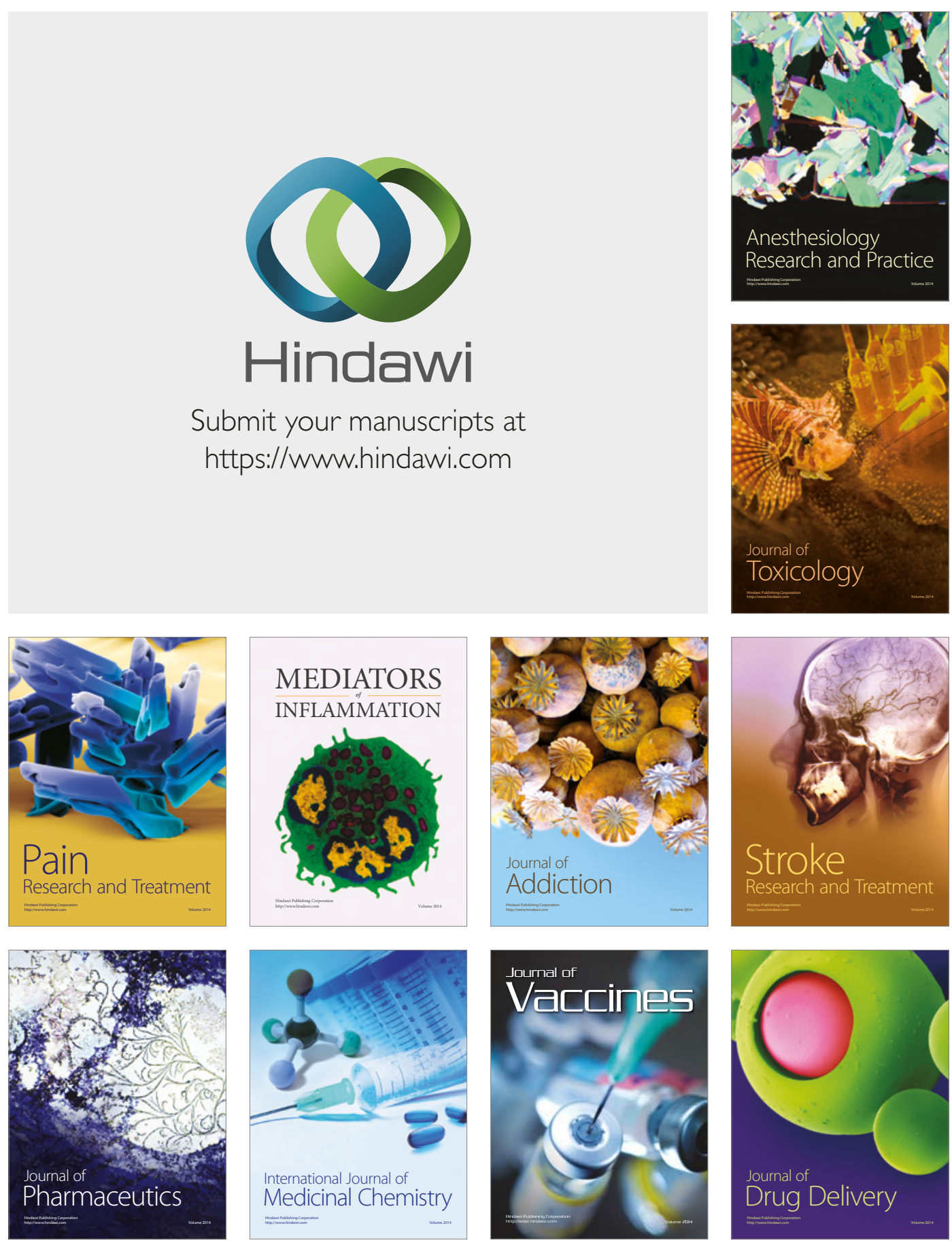